\title{
Negligible radiation protection of endothelial cells by vascular endothelial growth factor
}

\author{
MILA DONKER ${ }^{1,2}$, WOUTER R. VAN FURTH ${ }^{2}$, SACHA MULDER-VAN DER KRACHT ${ }^{1,2}$, \\ KOOS E. HOVINGA ${ }^{1,2}$, JOOST J.C. VERHOEFF ${ }^{1,2}$, LUKAS J.A. STALPERS ${ }^{1}$ and CHRIS VAN BREE ${ }^{1}$ \\ ${ }^{1}$ Laboratory of Experimental Oncology and Radiobiology (LEXOR), Department of Radiation Oncology; \\ ${ }^{2}$ Department of Neurosurgery, Academic Medical Center, University of Amsterdam, Amsterdam, The Netherlands
}

Received April 3, 2007; Accepted May 22, 2007

\begin{abstract}
Glioblastoma multiforme (GBM) is a radioresistant tumor. Tumor neoangiogenesis is an important mechanism for tumor sustenance. Angiogenesis is primarily mediated by vascular endothelial growth factor (VEGF), and earlier studies have suggested that VEGF protects human umbilical vein endothelial cells (HUVECs) against high doses of radiation. We tried to extend these findings to other endothelial cell lines and clinically relevant irradiation doses. Therefore, four different endothelial cell lines (HUVEC-C, primary HUVEC-P, an immortalized HUVEC cell line: EC-RF24, and bovine retina endothelial cells: BREC) were cultured without or with recombinant human $\operatorname{VEGF}_{165}\left(\mathrm{rhVEGF}_{165}\right)$. Cells were irradiated with $\gamma$-rays from a ${ }^{137} \mathrm{Cs}$-source. Radiosensitivity was determined by proliferation or clonogenic assay. Apoptosis was assayed by flow cytometric determination of the sub-G1 population or by counting nuclear fragmentation. We found that the biologically active $\operatorname{rhVEF}_{165}$ was able to improve clonogenic survival of HUVEC-C after 2 and 5 Gy. However, rhVEGF $_{165}$ could not significantly alter the radiosensitivity of all cell lines studied in proliferation assays. $\mathrm{rhEGF}_{165}$ only slightly reduced apoptosis in HUVEC-C after $3 \mathrm{~Gy}$. In conclusion, the radioprotective effect from rhVEGF $_{165}$ was found on different endothelial cell lines after clinically relevant radiation doses was negligible. We therefore hypothesize that the high VEGF-levels found in GBM in vivo do not reduce the radiosensitivity of endothelial cells, which is thought to contribute to the strong radioresistance of the tumor vasculature.
\end{abstract}

Correspondence to: Dr Chris van Bree, LEXOR, Department of Radiation Oncology, AMC-UvA, Room F0-205, Meibergdreef 9, 1105 AZ Amsterdam, The Netherlands

E-mail: c.vanbree@amc.uva.nl

Key words: glioblastoma multiforme, vascular endothelial growth factor, endothelial cells, ionizing radiation, radiosensitivity

\section{Introduction}

Glioblastoma multiforme (GBM) is the most common and most aggressive primary brain tumor. Most patients die within 2 years and the 5-year survival rate has remained at $4-5 \%$ for the last 30 years, in spite of the many improvements made in diagnostic and treatment possibilities, such as neurosurgery, neuro-imaging and radiotherapy $(1,2)$. The current standard of care for patients with a malignant glioma is resection followed by radiotherapy and chemotherapy (3). Unfortunately, this tumor is very resistant to radiotherapy and local tumor recurrence occurs in $\sim 90 \%$ of patients. The mechanisms of the extremely high radioresistance of GBM is largely unknown, but it is very likely that the well-developed vascular system has an important role in tumor sustenance. Garcia-Barros et al for example found that tumors grown in asmase deficient mice grew faster and showed lower levels of apoptosis in endothelial cells after irradiation (4).

Tumor growth in general and fast growing tumors such as GBM in particular are thought to be dependent on angiogenesis by sprouting of new vessels from pre-existing vasculature (5). This process is regulated by inducers and inhibitors released from tumor cells, endothelial cells and the extracellular matrix. Vascular endothelial growth factor (VEGF) is the bestcharacterized growth factor that induces vessel formation. VEGF increases vascular permeability and stimulates vessel formation by recruiting progenitor endothelial cells from the bone marrow (6). VEGF-function is suggested to be primarily restricted to endothelial cells, because it binds to two endothelial cell tyrosine kinase receptors, VEGFR1 and VEGFR2 (7). VEGF-expression is regulated by a variety of growth factors (8). Moreover, ionizing radiation (IR) has been shown to induce VEGF-transcripts and a dose-dependent upregulation of VEGF-expression in several tumor cell lines (7,9-11). VEGF has been implicated to protect endothelial cells from radiation-induced cell death $(7,9,12-14)$. A current hypothesis is that GBM-derived VEGF, probably elevated after irradiation, is a radioprotector for endothelial cells, thereby contributing to the radioresistance of GBM (11). Most of the earlier studies used HUVEC-C as the endothelial cell line to assess the radioprotective properties of VEGF. However, according to Charalambous et al (15), even normal brain endothelial cells and GBM endothelial cells have significant 
phenotypic and functional distinctions. In addition, the protection of endothelial cells has been reported after relatively high radiation doses $(7,9,12)$. However, it has been noted that proliferation of human coronary artery endothelial cells was not significantly altered by VEGF after $2 \mathrm{~Gy}$, while it was enhanced after $10 \mathrm{~Gy}$ (13). The role of the endothelium in the response of solid tumors after a high single dose ( $>8 \mathrm{~Gy})$ as well as after fractionated irradiation (1.8-3 Gy per fraction) is still a matter of debate (16). Therefore, we investigated whether VEGF also protects other endothelial cells from radiation at clinically relevant doses. For this purpose, we studied the effects of rhVEGF $_{165}$ and irradiation on HUVEC-C, endothelial cells Ruud Fontijn 24 (EC-RF24) which is an immortalized cell line of primary HUVECs, primary human umbilical vein endothelial cells (HUVEC-P) and bovine retina endothelial cells (BREC), which are more closely related to brain endothelial cells (17).

\section{Materials and methods}

Cell culture. Endothelial cells Ruud Fontijn 24 (EC-RF24), a primary HUVEC cell line immortalized using an amphotrophic, replication-deficient retrovirus containing theE6/E7 genes of human papilloma virus (a gift from Dr R. Fontijn and Dr J.V. van Thienen, Department of Biochemistry, AMC, Amsterdam), were maintained in medium 199 + 25 mM Hepes (Gibco Life Technologies, Paisley, Scotland), supplemented with $20 \%$ heat-inactivated fetal calf serum, $2 \%$ glutamine, penicillin $(100 \mathrm{IU} / \mathrm{ml})$ and streptomycin $(100 \mu \mathrm{g} / \mathrm{ml}), 0.5 \%$ heparin (Sigma-Aldrich Chemie BV, Zwijndrecht) and between 6-25 $\mu \mathrm{g} / \mathrm{ml}$ endothelial cell growth factor (ECGS) from Sigma, called complete growth medium (18). Freshly isolated primary HUVEC (HUVEC-P) were a kind gift from Dr R. Nieuwland and Dr D.P.G. Snoeck (Department of Clinical Chemistry, AMC). HUVEC-P were maintained in medium 199, supplemented with $10 \%$ human serum, $1 \%$ Lglutamine, penicillin (100 IU/ml), streptomycin $(100 \mu \mathrm{g} / \mathrm{ml})$, $0.2 \%$ fungizone, $0.5 \%$ EGF, $1 \%$ FGF and $0.1 \%$ heparin, called complete growth medium. HUVEC-C were obtained from Cambrex and were grown in fully supplemented endothelial cell basal medium (EBM)-2 medium as recommended by the manufacturer. The bovine retina endothelial cells (BREC) were kindly donated by Dr R.O. Schlingemann (Department of Ophthalmology, AMC) and were maintained in Dulbecco's modified Eagle's medium (DMEM) (Gibco Life Technologies), supplemented with $10 \%$ heat-inactivated fetal calf serum and $2 \%$ glutamine, penicillin $(100 \mathrm{IU} / \mathrm{ml})$ and streptomycin $(100 \mu \mathrm{g} / \mathrm{ml})$, called complete growth medium. All plates and flasks used to maintain EC-RF24, BREC and HUVEC-P were coated for $30 \mathrm{~min}$ with fibronectin $1 \mathrm{mg} / \mathrm{ml}$ PBS (16).

Reagents. Recombinant human $\operatorname{VEGF}_{165}\left(\mathrm{rhVEGF}_{165}\right)$ was obtained from R\&D Systems (Abingdon, UK) and dissolved in phosphate-buffered saline containing at least $0.1 \%$ bovine serum albumin.

Reverse transcription-polymerase chain reaction (RT-PCR). Total RNA was isolated from $\sim 10^{6}$ cells using the High Pure RNA isolation kit (Roche Applied Science, Mannheim,
Germany). Total RNA (50 ng) was subjected to RT-PCR using the One-Step RT-PCR kit (Qiagen Benelux, Venlo, The Netherlands) with primers for VEGFR1 (forward: 5'-TG CTTGAAACCGTAGCTGG-3' and reverse: 5'-GGTGCCA GAACCACTTGATT-3', Tm $=60^{\circ} \mathrm{C}, 30$ cycles), for VEGFR2 (forward: 5'-CCTGGAGCTGAGAACTACCG-3'; reverse: 5'-GCTTTCTGAGAAGACCACCG-3'; Tm $=53^{\circ} \mathrm{C}$, 35 cycles) and for GAPDH (forward: 5'-GGTGGAGGTCGGAGTC AACGGA-3'; reverse: 5'-GAGGGATCTCGCTCCTGGAG GA-3'; Tm $=62^{\circ} \mathrm{C}, 25$ cycles). Products were electrophoresed on a $1 \%$ agarose containing ethidium bromide. PCR-products were visualized using a ChemiDoc XRS system with Quantity One $^{\circledR}$ (v4.4) software (BioRad Laboratories, Hercules, CA).

Western blotting. The mitogen-activated protein kinase (MAPK) pathway exists in all eukaryotes. It consists of several subgroups including ERK 1/2 (extracellular signalregulated kinase). These kinases are a major determinant in the control of diverse cellular processes such as proliferation, apoptosis, survival and differentiation. They are activated by diverse stimuli including cytokines and growth factors and can be used as an indication to cell proliferation in response to growth factors such as VEGF (19). HUVEC-C and HUVEC-P were serum starved for $18 \mathrm{~h}$ after which rhVEGF $_{165}$ (10 ng/ml) was added. After washing with ice-cold PBS, whole cell lysates were prepared at various time points. Proteins were separated on $15 \%$ SDS-PAGE and were transferred to polyvinylidine difluoride membranes using semi-dry electroblotting. The blots were stained for ERK1/2 (phosphospecific (Thr202/Tyr204) mouse monoclonal antibody (E10), Cell Signaling Technology, Danvers, MA) and B-actin (mouse monoclonal AC-15, Sigma-Aldrich, Zwijndrecht, The Netherlands) and a secondary HRP-conjugated goat antimouse antibody (Dako, Glostrup, Denmark). Standard enhanced chemiluminescence was used to visualize the protein using a ChemiDoc XRS system with Quantity One (v4.4) software (BioRad Laboratories).

Radiation treatment. Irradiation was performed with $\gamma$-rays from a ${ }^{137} \mathrm{Cs}$-source at a dose rate of $\sim 0.8$ Gy per minute.

Clonogenic assay. Clonogenic assays were performed according to Gorski et al (9). HUVEC-C were plated in appropriate densities in fully supplemented EBM-2 medium. Eighteen hours after plating, the medium was replaced with serum-free medium without additional growth factors, to which a defined amount (0-100 ng/ml) of rhVEGF $_{165}$ had been added. Four hours later, the cells were irradiated with 0,2 or 5 Gy. The cells were incubated for $48 \mathrm{~h}$, after which medium was replaced with complete EGM-2 containing $10 \mathrm{ng} / \mathrm{ml}$ rhVEGF $_{165}$. After 12-14 days, the cells were fixed with $6 \%$ glutaraldehyde and stained with $0.05 \%$ crystal violet. Colonies containing $>50$ cells were scored as originating from one surviving cell counted and surviving fractions were calculated as compared to control (plating efficiency was $11 \pm 0.5 \%$ ).

Proliferation assay. Proliferation assays were performed according to Gorski et al (9). In short, cells were plated in 96-well plates at a concentration of 1000 cells in $0.1 \mathrm{ml}$ complete growth medium per well and allowed to attach 


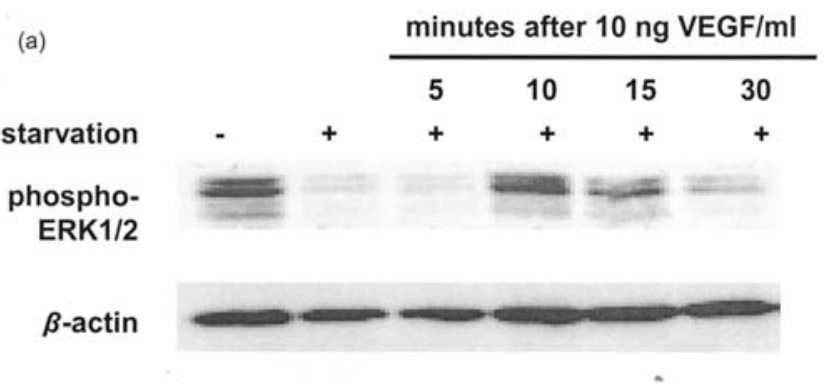

(b)

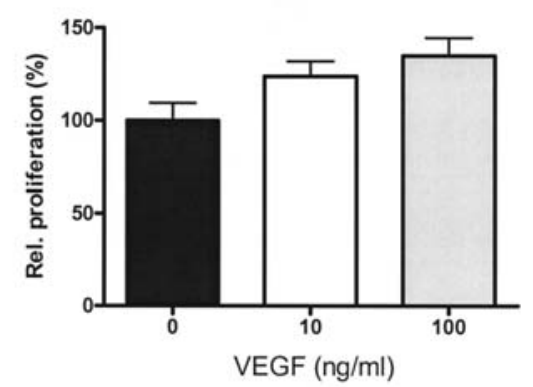

(c)

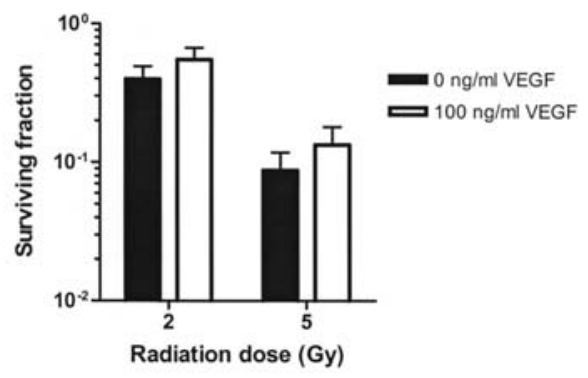

Figure 1. (a) A representative example of three separate Western blot experiments of VEGF-stimulated ERK activation in HUVEC-C. Non-starved and serum-starved cells were left untreated or stimulated with rhVEGF ${ }_{165}$ $(10 \mathrm{ng} / \mathrm{ml})$ for the indicated times. Cell lysates were analyzed by Western blotting with an phosphospecific monoclonal antibody against ERK1/2 and a monoclonal antibody against $\beta$-actin as a loading control. (b) Relative proliferation of HUVEC-C with 10 or $100 \mathrm{ng} / \mathrm{ml} \mathrm{rhVEGF}_{165}$ added to the complete growth medium normalized to the complete growth medium without additional VEGF. Means with standard errors of three separate experiments are shown. (c) Surviving fraction of HUVEC-C normalized to unirradiated controls with 0 or $100 \mathrm{ng} / \mathrm{ml} \mathrm{rhVEGF}_{165}$ added to serum-free medium $4 \mathrm{~h}$ prior to irradiation with 2 or $5 \mathrm{~Gy}$. Means with standard errors of at least three separate experiments are shown.

overnight. The next day, medium was removed and replaced with complete growth medium containing different concentrations of rhVEGF ${ }_{165}$. Four hours later, the plates were irradiated and $48 \mathrm{~h}$ after irradiation, the medium was replaced with complete growth medium. At $96 \mathrm{~h}$ after irradiation, the number of living cells was determined using an XTT-assay (Cell proliferation kit II, Roche Diagnostics, Mannheim, Germany).

Nicoletti DNA fragmentation assay. The Nicoletti DNA fragmentation assay is a flow cytometric method used for measuring the percentage of apoptotic nuclei after propidium iodide staining in hypotonic buffer, and thereby assessing apoptosis of specific cell populations in heterogeneous tissues (20). Induction of DNA fragmentation was performed similar to the co-culture experiments of Brown et al (14). HUVEC-C
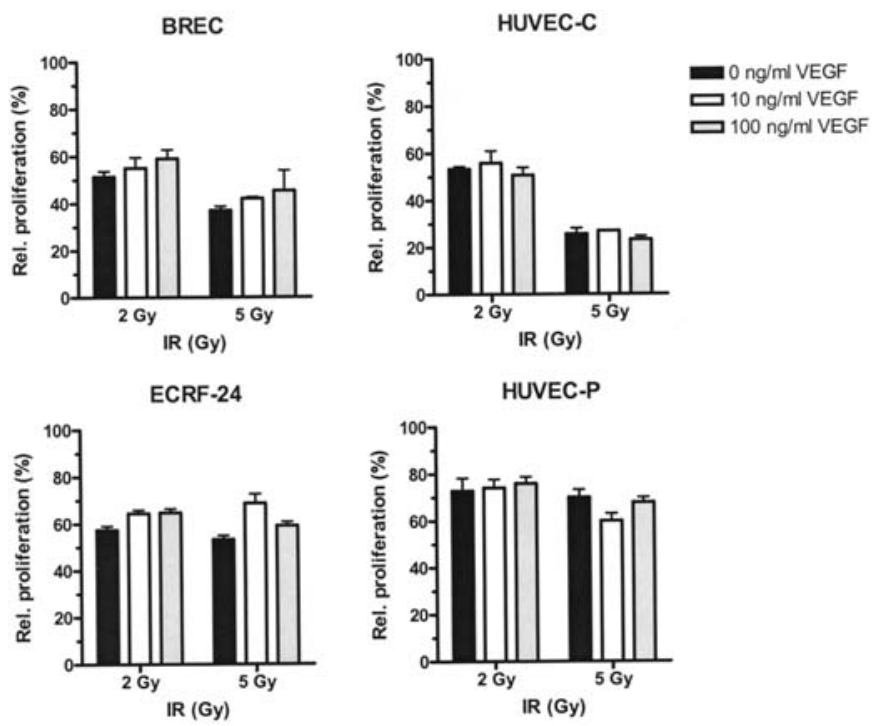

Figure 2. Proliferation of BREC, HUVEC-C, EC-RF24, and HUVEC-P without or with 10 or $100 \mathrm{ng} / \mathrm{ml} \mathrm{rhVEGF}_{165}$ in the complete growth medium after irradiation with 2 or 5 Gy normalized to unirradiated controls. Means with standard errors of at least three separate experiments are shown.

were plated in fully supplemented EBM-2 medium. Eighteen hours after plating, the medium was replaced with serum-free medium without additional growth factors, to which a defined amount $\left(0\right.$ or $100 \mathrm{ng} / \mathrm{ml}$ ) of $\mathrm{rhVEGF}_{165}$ had been added. Four hours later, the cells were irradiated with 0 or $3 \mathrm{~Gy}$ and incubated for another $24 \mathrm{~h}$. Both detached and attached cells were harvested and pelleted. The cells were resuspended in $200 \mu 1$ of hypotonic buffer $(0.1 \%$ sodium citrate, $0.1 \%$ Triton $\mathrm{X}-100,50 \mu \mathrm{g} / \mathrm{ml}$ propium iodide, dissolved in demi water) and stored for $24 \mathrm{~h}$ at $4^{\circ} \mathrm{C}$. Flow cytometric analysis was performed according to Nicoletti et al (20). The control consisted of nonirradiated HUVEC in fully supplemented medium without extra VEGF.

\section{Results}

We first determined whether the four cell systems used in this study responded to h VEGF $_{165}$ as described previously. Using RT-PCR, we found that VEGF receptors 1 and 2 were present in HUVEC-C, HUVEC-P and EC-RF24 (data not shown). Similar to $\mathrm{Yu}$ et al (21), we verified functional signal transduction in both HUVEC-C (Fig. 1a) and HUVEC-P by analyzing the phosphorylation of ERK1/2. After starvation, which is necessary to be able to follow the phosphospecific events induced by mitogenic stimuli, rhVEGF $_{165}$ induced a transient phosphorylation of ERK1/2 in both cell lines. We further determined the effect of $\mathrm{rhVGF}_{165}$ on proliferation of all cell lines studied. The addition of rhVEGF $_{165}$ slightly enhanced proliferation of HUVEC-C (Fig. 1b) which was also observed for HUVEC-P, EC-RF24 and BREC. Clonogenic radiation survival of HUVEC-C (Fig. 1c) was studied similarly to Gorski et al (9): VEGF was added in serum-free medium $4 \mathrm{~h}$ prior to irradiation and was replaced by complete growth medium $48 \mathrm{~h}$ after irradiation. The addition of $100 \mathrm{ng} \mathrm{rhVEF}_{165} / \mathrm{ml}$ slightly increased radiation survival.

Since HUVEC-P, EC-RF24 and BREC do not form colonies and do not survive prolonged periods $(>24 \mathrm{~h})$ 
(a)
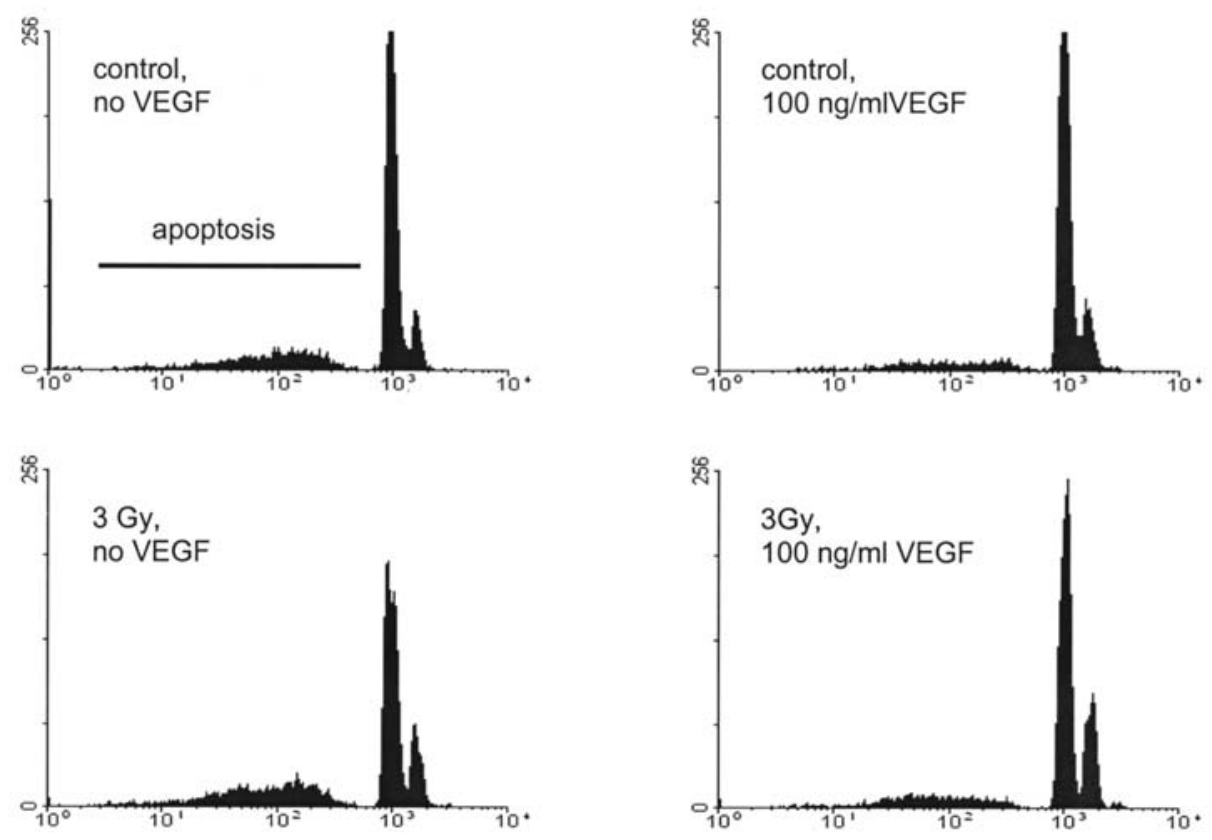

(b)

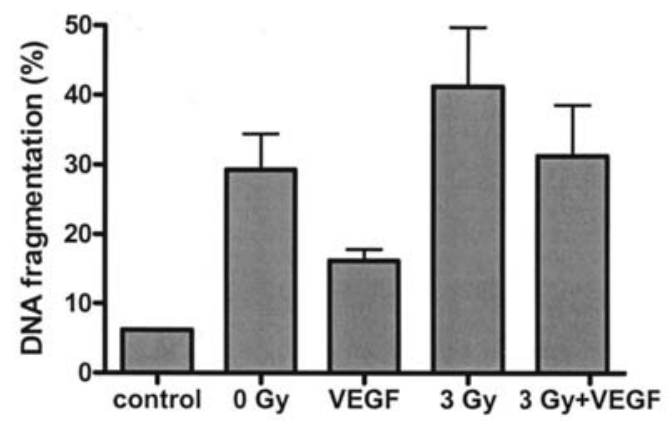

Figure 3. (a) A representative example of the flow cytometric analysis of DNA fragmentation of HUVEC-C without or with $100 \mathrm{ng} / \mathrm{ml}^{\mathrm{rhVEGF}} \mathrm{hb5}_{165}$ added to the serum-free medium before irradiation with 0 or $3 \mathrm{~Gy}$. (b) Quantification of the flow cytometric analysis. Means are shown with standard errors of at least three separate experiments. The control represents unirradiated HUVEC-C in complete growth medium without additional VEGF.

without serum, we performed proliferation assays to detect differences in radiosensitivity in the presence of additional rhVEGF $_{165}$ (Fig 2). Although in BREC and EC-RF24 there was a slight increase in proliferation when rhVEGF $_{165}$ was present before irradiation, it is evident that rhVEGF $_{165}$ did not alter radiosensitivity in HUVEC-P and HUVEC-C.

Since VEGF mediated protection has been attributed to a decreased induction of apoptosis (14), we performed experiments in which we added rhVEGF $_{165}$ to control and serum-starved HUVEC-C without and with irradiation with 3 Gy (Fig. 3). Serum starvation induced marked apoptosis $(29.3 \pm 4.2 \%)$ as compared to control cultures $(6.2 \pm 0.1 \%)$, which was reduced by $\operatorname{rhVEGF}_{165}(15 \pm 2 \%)$. Irradiation with 3 Gy provoked an increase in apoptosis of $\sim 12 \%$ as compared with the serum-starved unirradiated control. Although the presence of rhVEGF $_{165}$ reduced the level of apoptosis in serum-starved cells after $3 \mathrm{~Gy}$, it was not able to prevent an increase in apoptosis as compared to the serum-starved, nonirradiated rhVEGF $_{165}$ treated cultures (an increase of $\sim 15 \%$ ). Similar results were obtained when apoptosis was quantified as fragmented nuclei after staining with DAPI and scoring with a fluorescence microscope.

\section{Discussion}

In this study we show that h VEGF $_{165}$ has only a negligible effect on the radiosensitivity of four different endothelial cell lines. We checked the validity of our experimental set-up by confirming the presence of VEGF-receptors and a functional signal transduction upon stimulation with rhVEGF $_{165}$ as reported earlier (21). Moreover, we were able to show that rhVEGF $_{165}$ protects HUVEC-C from cell reproductive death 
as determined by clonogenic assay similarly to Gupta et al (7) and Gorski et al (9). When differences in radiation survival were expressed as mean inactivation doses (22), we found a similar radioprotective effect from $100 \mathrm{ng} \mathrm{VEGF} / \mathrm{ml}$ (dose modifying factor of 0.79). Brieger et al (23) did not find a significant increase in cell survival in a clonogenic assay after adding hhVEFF $_{165}$ to the medium of primary HUVEC. Unfortunately, we were not able to test our HUVEC-P in clonogenic assays. Our data support earlier findings that the level of protection induced by VEGF is more pronounced at higher radiation doses $(7,9)$. Also using thymidine uptake as a measure for proliferation, $100 \mathrm{ng} \mathrm{rhVEF}_{165} / \mathrm{ml}$ was unable to protect human coronary artery endothelial cells from $2 \mathrm{~Gy}$ of $\beta$ irradiation, while VEGF significantly improved proliferation after 10 Gy (13).

After confirming the radioprotection by rhVEGF $_{165}$ of HUVEC-C in clonogenic assays, we were curious whether rhVEGF $_{165}$ could protect other endothelial cells from radiation effects. Because of practical limitations we were forced to use proliferation assays to determine radiosensitivity, but we tried to use a similar experimental set-up as the clonogenic assays with respect to the additional $\mathrm{rhVEFF}_{165}$. Using proliferation as an endpoint, Gorski et al (9) and Brieger et al (23) have reported VEGF-mediated radioprotection. However, in our experiments, $\mathrm{rhVEGF}_{165}$ resulted in no or only in minor radioprotection of endothelial cell lines (HUVEC-P, ECRF-24, BREC), arguing against a prominent direct role of rhVEGF $_{165}$ in endothelial cell radioresistance.

Similar to our findings, there is ample evidence that rhVEGF $_{165}$ protects endothelial cells from apoptosis-inducing stimuli, such as preventing endothelial cells from apoptosis induced by serum starvation (7). With respect to VEGFmediated radioprotection, Brown et al (14) reported that in a co-culture set-up of HUVEC-C and U87, a radiation dose of 3 Gy yielded a significant increase of apoptosis from $8 \%$ (non-irradiated) to $36 \%$ (irradiated) which means an increase of $>400 \%$. We were able to induce an increase in fragmentation of only $\leq 140 \%$ after irradiation with 3 Gy which was not significantly altered when rhVEGF $_{165}$ was added. Others have also reported lower levels of apoptosis after a single dose of irradiation of endothelial cells. Hess et al (12) did not observe morphological signs of apoptosis or cleavage of caspase 3 in HUVEC after 10 Gy with or without VEGF. Bischof et al (24) found no significant increase in sub-G1 fraction in HUVEC and HDMEC (human dermal micro-vascular endothelial cells) after irradiation with 5 Gy. The reduction of radiation-induced apoptosis in the coculture set-up as described by Brown et al (14) might therefore be due to other factors than VEGF.

It has been postulated that after a high single dose of irradiation VEGF and bFGF protect endothelial cells from apoptosis by inhibiting the generation of ceramide (16). Fractionated irradiation (1.8-3 Gy per fraction) has been shown to activate hypoxia inducible factor-1 (HIF-1) which transactivates a number of genes, including VEGF (25). The HIF-1 target genes may inhibit endothelial cell apoptosis contributing to tumor radioresistance (16). Our data indicate that VEGF only marginally protects endothelial cells in culture from a single radiation dose of $\leq 5 \mathrm{~Gy}$, suggesting that other factors may be more important. However, we can not exclude the possibility that radioresistance of endothelial cells in vivo is influenced by VEGF. This is underscored by recent evidence that endothelial cells provide a niche in vivo for glioma stem cells (26). Since glioma stem cells are more radioresistant than glioma cells without stem cell markers (27), it is evident that the targeting of tumor vasculature is still an attractive modality to combine with radiotherapy.

\section{Acknowledgements}

We wish to thank Dr R. Fontijn, Dr J.V. van Thienen, Dr R. Nieuwland, Dr D.P.G. Snoeck and Dr R.O. Schlingemann for kindly providing materials and cell lines. Ing. H.M. Rodermond is acknowledged for expert technical assistance and Dr N.A.P. Franken and Professor J.P. Medema for critically reviewing the manuscript. The Maurits and Anna de Kock foundation is acknowledged for sponsoring laboratory equipment. This study was financially supported by the J.A. Cohen Institute (IRS grant \# 7.1.7.).

\section{References}

1. McLendeon RE and Halperin EC: Is the long-term survival of patients with intracranial glioblastoma multiforme overstated? Cancer 98: 1745-1748, 2003.

2. Behin A, Hoang-Xuan K, Carpentier AF and De Lattre JY: Primary brain tumours in adults. Lancet 361: 323-331, 2003.

3. Stupp R, Hegi ME, van den Bent MJ, et al: Changing paradigms an update on the multidisciplinary management of malignant glioma. Oncologist 11: 165-180, 2006.

4. Garcia-Barros M, Paris F, Cordon-Cardo C, et al: Tumor response to radiotherapy regulated by endothelial cell apoptosis. Science 300: 1155-1159, 2003.

5. Ferrara $\mathrm{N}$ and Davis-Smyth T: The biology of vascular endothelial growth factor. Endocr Rev 18: 4-25, 1997.

6. Toi M, Matsumoto T and Bando H: Vascular endothelial growth factor: its prognostic, predictive, and therapeutic implications. Lancet Oncol 2: 667-673, 2001.

7. Gupta VK, Jaskowiak NT, Beckett MA, et al: Vascular endothelial growth factor enhances endothelial cell survival and tumor radioresistance. Cancer J 8: 47-54, 2002.

8. Ferrara N, Gerber HP and LeCouter J: The biology of VEGF and its receptors. Nat Med 9: 669-676, 2003.

9. Gorski DH, Beckett MA, Jaskowiak NT, et al: Blockage of the vascular endothelial growth factor stress response increases the antitumor effects of ionizing radiation. Cancer Res 59: 3374-3378, 1999.

10. Lund EL, Hog A, Olsen MW, Hansen LT, Engelholm SA and Kristjansen PE: Differential regulation of VEGF, HIF1alpha and angiopoietin-1, -2 and -4 by hypoxia and ionizing radiation in human glioblastoma. Int J Cancer 108: 833-838, 2004.

11. Hovinga KE, Stalpers LJ, van Bree C, et al: Radiation-enhanced vascular endothelial growth factor (VEGF) secretion in glioblastoma multiforme cell lines - a clue to radioresistance? J Neurooncol 74: 99-103, 2005.

12. Hess C, Vuong V, Hegyi I, et al: Effect of VEGF receptor inhibitor PTK787/ZK222584 [correction of ZK222548] combined with ionizing radiation on endothelial cells and tumour growth. Br J Cancer 85: 2010-2016, 2001.

13. Kermani P, Leclerc G, Martel R and Fareh J: Effect of ionizing radiation on thymidine uptake, differentiation, and VEGFR2 receptor expression in endothelial cells: the role of $\operatorname{VEGF(165).~}$ Int J Radiat Oncol Biol Phys 50: 213-220, 2001.

14. Brown CK, Khodarev NN, Yu J, et al: Glioblastoma cells block radiation-induced programmed cell death of endothelial cells. FEBS Lett 565: 167-170, 2004.

15. Charalambous C, Hofman FM and Chen TC: Functional and phenotypic differences between glioblastoma multiforme-derived and normal human brain endothelial cells. J Neurosurg 102: 699-705, 2005.

16. Fuks $\mathrm{Z}$ and Kolesnick R: Engaging the vascular component of the tumor response. Cancer Cell 8: 89-91, 2005. 
17. Antonetti DA, Barber AJ, Hollinger LA, Wolpert EB and Gardner TW: Vascular endothelial growth factor induces rapid phosphorylation of tight junction proteins occludin and zonula occluden 1. A potential mechanism for vascular permeability in diabetic retinopathy and tumors. J Biol Chem 274: 23463-23467, 1999.

18. Diekman MJ, Zandieh Doulabi B, Platvoet-Ter Schiphorst M, Fliers E, Bakker O and Wiersinga WM: The biological relevance of thyroid hormone receptors in immortalized human umbilical vein endothelial cells. J Endocrinol 168: 427-433, 2001.

19. Murphy LO and Blenis J: MAPK signal specificity: the right place at the right time. Trends Biochem Sci 31: 268-275, 2006.

20. Nicoletti I, Migliorati G, Pagliacci MC, Grignani F and Riccardi C: A rapid and simple method for measuring thymocyte apoptosis by propidium iodide staining and flow cytometry. J Immunol Methods 139: 271-279, 1991.

21. Yu Y and Sato JD: MAP kinases, phosphatidylinositol 3-kinase, and p70 S6 kinase mediate the mitogenic response of human endothelial cells to vascular endothelial growth factor. J Cell Physiol 178: 235-246, 1999.
22. Fertil B, Dertinger H, Courdi A and Malaise EP: Mean inactivation dose: a useful concept for intercomparison of human cell survival curves. Radiat Res 99: 73-84, 1984.

23. Brieger J, Schroeder P, Gosepath J and Mann WJ: VEGFsubtype specific protection of SCC and HUVECs from radiation induced cell death. Int J Mol Med 15: 145-151, 2005.

24. Bischof M, Abdollahi A, Gong P, et al: Triple combination of irradiation, chemotherapy (pemetrexed), and VEGFR inhibition (SU5416) in human endothelial and tumor cells. Int J Radiat Oncol Biol Phys 60: 1220-1232, 2004.

25. Moeller BJ, Cao Y, Li CY and Dewhirst MW: Radiation activates HIF-1 to regulate vascular radiosensitivity in tumors: role of reoxygenation, free radicals, and stress granules. Cancer Cell 5: 429-441, 2004.

26. Calabrese C, Poppleton H, Kocak M, et al: A perivascular niche for brain tumor stem cells. Cancer Cell 11: 69-82, 2007.

27. Bao S, Wu Q, McLendon RE, et al: Glioma stem cells promote radioresistance by preferential activation of the DNA damage response. Nature 444: 756-760, 2006. 